\title{
Identificação dos fatores ambientais que influenciam a funcionalidade dos utilizadores de cadeira de rodas
}

\author{
Identification of environmental \\ factors which influence wheelchair \\ users' functioning
}

FisiSenectus. Unochapecó Ano 2, n. 2 - Jul/Dez. 2014 p. $19-33$

Teresa Margarida Marques Alves. ft.teresaalves@gmail.com IPC-ESTeSC Coimbra Health School, Dept de Fisioterapia.

Anabela Correia Martins. anabelacmartins@estescoimbra.pt

IPC-ESTeSC Coimbra Health School, Dept de Fisioterapia.

\section{Resumo}

Introdução: A Classificação Internacional de Funcionalidade, Incapacidade e Saúde (ICF) foi aprovada pela OMS em 2001 e aborda funções e estruturas, atividades e participação e fatores contextuais (fatores ambientais e pessoais). 0 componente fatores ambientais é constituído por cinco capítulos: 1. produtos e tecnologia, 2. ambiente natural e mudanças ambientais feitas pelo homem, 3. apoios e relacionamentos, 4. atitudes e 5. serviços, sistemas e políticas. As intervenções devem focar no ambiente físico, nas tecnologias de apoio, na promoção de saúde e nos programas sociais com base na igualdade para facilitar a participação social dos utilizadores de cadeira de rodas (CDR). A CDR é uma tecnologia de apoio específica que pode aumentar significativamente a mobilidade e a participação social dos seus utilizadores. Os fisioterapeutas atuam em vários níveis com o objetivo de promover a funcionalidade, a independência e prevenir a incapacidade. Objetivo: Determinar as categorias do componente fatores ambientais da ICF que influenciam a funcionalidade no domicílio e na comunidade/sociedade, considerando a perceção dos utilizadores de CDR. Materiais e métodos: Utilizou-se o Método Delphi, um método qualitativo para coleta de informação como base para planeamento e tomada de decisões. Corresponde a um processo comunicativo com: intervenção de experts, utilização de um questionário formal, anonimato de respostas, feedback das respostas do grupo, múltiplas repetições do questionário e participação informada, com o objetivo de transformar opiniões em consensos de grupo. Conclusão: É necessário adequar os fatores externos ao indivíduo para que este possua máxima funcionalidade e segurança para uma participação ativa e efetiva na sociedade em que se insere.

\section{Palavras-chave}

Classificação Internacional de Funcionalidade, Incapacidade e Saúde; Fatores ambientais; Fisioterapia; Funcionalidade; Cadeira de rodas.

\section{Fisi enectus}




\begin{abstract}
Introduction: The International Classification of Functionality, Disability and Health (ICF) was approved by the WHO in 2001 and considers functions and structures, activities and participation as well as contextual factors (environmental and social factors). The environmental factors component is composed by five chapters: 1.products and technology, 2.natural environment and human-made changes to environment, 3.support and relationships, 4.attitudes, 5.services, systems and policies. To facilitate the wheelchair users' social participation efforts should be focused at the physical environment, support technology-related interventions, health promotion and in equality based social programs. The wheelchair is a specific support technology that may meaningfully increase the mobility and social participation of their users. Physiotherapists act in several levels to promote functionality and independence while preventing disability. Objective: To determinate the ICF environmental factors component categories that influence the functionality in the residences and in the community/society, based on wheelchair users' perception. Methods: The Delphi method was used, a qualitative method to gather information for planning and decision making. It corresponds to a communicative process with: expert intervention, use of formal questionnaire, anonymity answers, group's answers feedback, multiple repetitions of the questionnaire and informed participation, with the aim of converting opinions into a group consensus. Conclusion: It is necessary do fit the external factors to the person so that its functionality and safety are maximized, thereby increasing its effective participation in society.
\end{abstract}

\title{
Keywords
}

International Classification of Functioning, Disability and Health, Environmental Factors, Physiotherapy, Functioning, Wheelchair

\section{Introdução}

$\infty \times \infty \times \infty \times \infty \times \infty \times \infty \times \infty \times \infty \times \infty \times \infty \times \infty \times \infty \infty \infty$

A Organização Mundial de Saúde (OMS) é responsável pela realização de classificações internacionais de saúde. Estas representam modelos consensuais a serem incorporados pelos sistemas de saúde, gestores e usuários, visando a utilização de uma linguagem comum para a descrição de condições, problemas ou intervenções na área da saúde ${ }^{1}$. A Classificação Internacional de Funcionalidade, Incapacidade e Saúde, (ICF), foi desenvolvida pela OMS e foi aprovada em maio de $2001^{2}$. Surgiu como revisão da Classificação Internacional de Deficiência, Incapacidade e Handicap (ICIDH-2). Nessa revisão o nome e a ênfase negativa dos termos deficiência, incapacidade e handicap foram alterados para descrições neutras das estruturas, funções, atividades e participação. Outra alteração foi a inclusão dos fatores ambientais como parte da classificação ${ }^{3}$.

A ICF confere uma conceitualização completa e consistente relativa à incapacidade e à funcionalidade, evitando as falácias de que a incapacidade acontece apenas como consequência médica ou apenas socialmente criada $^{4}$.

Neste trabalho de investigação são considerados apenas os fatores ambientais, mas tendo sem- pre presente que estes não atuam isoladamente das outras componentes da ICF IC $^{5}$ Os fatores ambientais referem-se ao meio físico, social e comportamental onde os indivíduos se inserem ${ }^{6}$. Esse componente é constituído por cinco capítulos: 1 . Produtos e tecnologia; 2. Ambiente natural e mudanças ambientais feitas pelo homem; 3. Apoios e relacionamentos; 4. Atitudes; e 5 . Serviços, sistemas e políticas ${ }^{2}$.

Os fatores ambientais são determinantes cruciais da participação dos indivíduos nas atividades da vida diária7. Ou seja, o contexto ambiental, os ambientes domiciliário e comunitário em que os indivíduos se inserem influenciam a participação ${ }^{8}$. 0 constructo básico do componente Fatores Ambientais é o impacto facilitador ou limitador das características do mundo físico, social e atitudinal. A influência dos fatores ambientais classifica-se de acordo com o primeiro qualificador como "barreira" ou "facilitador"2. Desse modo, relacionam-se com as condições de saúde, criando incapacidade ("barreira") ou restaurando a funcionalidade ("facilitador"), dependendo da influência que determinado fator exerce ${ }^{3}$. Esse modelo social de incapacidade foi incorporado pela ICF e tem vindo a acentuar a consciência de que as barreiras à participação e integração são as causas por excelência do desenvolvimento de incapacidade ${ }^{9,10}$. 
As diferenças entre culturas na interpretação da incapacidade demonstram que a vida das pessoas com incapacidade é limitada não tanto pelo tipo específico de incapacidade, mas pela interpretação social desta ${ }^{11}$. Um dos objetivos do fisioterapeuta é contribuir para a promoção da funcionalidade e independência, bem como prevenir a incapacidade ${ }^{5}$. Os níveis de funcionalidade e incapacidade, nos contextos relevantes, são definidos pelas capacidades físicas do indivíduo e pela sua interação com elementos físicos, sociais e culturais do ambiente ${ }^{12}$.

As barreiras físicas, sociais e atitudinais na sociedade são um componente importante na limitação das atividades e nas restrições da participação da pessoa, paralelamente à deficiência. Assim, quebrar as práticas sociais incapacitantes torna-se tão importante como um processo associado à minimização da deficiência. Portanto, reconhecer a influência dos fatores ambientais no desempenho de cada indivíduo no seu ambiente natural e quotidiano assume uma função essencial em qualquer intervenção ${ }^{2,13}$.

A funcionalidade/incapacidade trata-se então de uma experiência multidimensional, influenciada por todos os componentes da ICF ${ }^{14,15}$ e resulta da relação complexa entre a condição de saúde e os fatores contextuais (fatores ambientais e pessoais) $)^{2}$.

Com o intuito de desenvolver padrões de participação mais ativos na sociedade das pessoas utilizadoras de cadeiras de rodas (CDR) torna-se necessário adequar ou modificar os ambientes. Esses indivíduos são os que melhor sabem detetar as barreiras físicas, sociais, atitudinais, e relativas a produtos e tecnologias. São assim fontes de informação relevante para a adaptação dos ambientes em questão, garantindo a sua funcionalidade e autonomia, bem como a de todos que os utilizarem ${ }^{16}$.

Para facilitar a participação social dos utilizadores de CDR deve-se focar não apenas no ambiente físico, mas também em outros aspetos relacionados: as intervenções relacionadas com tecnologia de apoio (com base no modelo universal), os programas de assistência pessoal, promoção de saúde e de forma física e os programas que melhorem o civismo, atentando principalmente nos fatores sociais com base na igualdade ${ }^{17}$. Por sua vez, a análise do ambiente é um modo útil de examinar o efeito do ambiente social na saúde e estados relacionados ${ }^{18}$.

A CDR é uma tecnologia de apoio específica que pode aumentar significativamente a mobilidade e a participação social dos seus utilizadores. Para que isso seja possível é necessário que a CDR seja apropriada e adaptada às necessidades de cada indivíduo sob pena de poder ser interpretada como barreira à participação ${ }^{19}$. A nova geração de CDR pode ser ajustada para permitir ao utilizador melhor adaptação, melhor postura e características de propulsão modificáveis. A responsabilidade de otimizar essa tecnologia é dos profissionais de saúde, tendo sempre em consideração a complexidade da interação entre o utilizador, a CDR e as características ambientais $^{1,20}$. A escolha da tecnologia de apoio deve basear-se, tanto nas necessidades como nas preferências. A tecnologia nunca deve ser 0 ponto de partida, o qual deve ser as necessidades, desejos e objetivos do utilizador de forma a otimizar a sua posterior utilização $0^{21}$.

A Reabilitação Baseada na Comunidade (RBC) existe como estratégia de desenvolvimento comunitário para a reabilitação, igualdade de oportunidades e inclusão de todas as pessoas com incapacidade, promovendo a sua plena participação ${ }^{22}$. A participação requer acesso. A identificação de barreiras e soluções físicas, sociais, culturais, econômicas e atitudinais requer a existência de legislação para facilitar acesso, participação e inclusão ${ }^{23}$. A RBC promove os direitos e a participação ativa das pessoas com incapacidade e desenvolve esforços para garantir que todas as pessoas com incapacidade exerçam os mesmos direitos e oportunidades que os outros membros da sociedade. Ou seja, a sua inclusão ${ }^{24}$.

Nesse contexto conceitual é indispensável repensar as intervenções. Deve-se proceder à adoção de uma abordagem que se preocupe não apenas com indivíduos que experienciam vários tipos de incapacidade, mas também relacionada com a sociedade e as suas várias estruturas físicas, sociais e atitudinais. 0 objetivo primordial de um programa de reabilitação será então a remoção metódica de barreiras ${ }^{24}$. 
No mesmo sentido, surge o conceito de empowerment, cujo alicerce é a ideia de que as pessoas com incapacidade têm o direito fundamental de tomar decisões informadas sobre os cuidados de saúde a que são submetidas ${ }^{1}$. A sua importância relaciona-se com a educação da comunidade sobre os direitos que cada indivíduo com incapacidade tem para tomar decisões sobre a sua própria vida. 0 conceito é abrangente e inclui aspetos sociais, políticos e culturais e é alcançado por meio da colaboração entre profissionais de saúde (mais especificamente fisioterapeutas) e os indivíduos. Para que esse conceito possa ser aplicado corretamente tem de haver uma abordagem centrada no indivíduo resultante da interação entre o raciocínio clínico, valores, virtudes e conhecimento do fisioterapeuta ${ }^{25}$.

Dentre os objetivos, podemos mencionar: tornar os indivíduos autônomos, aumentar a confiança na autoeficácia, envolve-los no processo de intervenção, evitar modalidades passivas, promover o desenvolvimento de estratégias de ajustamento e adaptação, permitir melhor continuidade de serviços, bem como de cuidados mais competentes e planos de cuidados de saúde mais individualizados $^{24}$. 0 crescimento econômico não produzirá melhorias na saúde e educação se não estiver inerente à utilização do conceito empowerment. As alterações das condições sociais com intuito de assegurar a liberdade das pessoas, aumentar a sua autonomia e fazê-las tomar as suas próprias decisões, conduzem a reduções marcadas nas desigualdades em saúde ${ }^{27}$.

Com este estudo pretendeu-se determinar as categorias relativas ao componente fatores ambientais da ICF que influenciam a funcionalidade no domicílio e na comunidade/sociedade, considerando a percepção das pessoas utilizadoras de CDR. A influência desses fatores classificou-se como barreira ou facilitador de acordo com o primeiro qualificador da referida classificação.

\section{Materiais e métodos}

$\infty \times \infty \times \infty \times \infty \times \infty \times \infty \times \infty \times \infty \times \infty \times \infty \times \infty \times \infty \times \infty)$

Neste trabalho de investigação utilizou-se o Método Delphi. É um método qualitativo para coleta de informação e é utilizado como base para um planeamento e tomada de decisões. Corresponde a um processo comunicativo com várias características fundamentais: intervenção de experts, utilização de um questionário formal, anonimato de respostas, feedback das respostas do grupo, múltiplas repetições do questionário e participação informada com o objetivo de transformar opiniões em consensos de grupo. 0 uso de experts é fundamental, devido ao domínio de informação, conhecimento específico e pormenorizado do tema abordado, o que maximiza o número e representatividade das ideias e opiniões reunidas, minimizando o número de pessoas que seria necessário questionar ${ }^{5}$.

Neste estudo os experts são os próprios utilizadores, há pelo menos um ano, de CDR integrados na comunidade, onde desempenham um papel ativo e uma atividade profissional ativos.

Participaram 16 indivíduos utilizadores de CDR com idades entre os 29 e 60 anos, residentes em cidades ou aldeias do interior e litoral do país.

Todos os procedimentos de investigação foram realizados de acordo com os princípios éticos contidos na Declaração de Helsinque e obteve-se um termo de consentimento informado de todos os participantes

\section{Resultados}

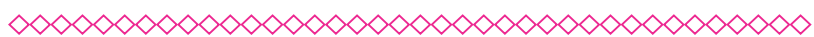

A aplicação do método Delphi foi realizada em três fases.

\section{Fase 1}

A primeira fase corresponde ao questionário efetuado aos experts, é constituída por três partes e culmina na transcrição de conceitos relevantes para categorias da ICF.

Na primeira parte questionou-se sobre os principais fatores ambientais que mais influenciam a sua funcionalidade. Essa fase correspondeu a uma questão aberta orientada pelo enunciado onde se explicava, sucintamente, a que correspondia o conceito de fatores ambientais. Essa parte corresponde à primeira fase de recolha de dados. 
As respostas recolhidas foram transcritas para conceitos significativos, o que corresponde à segunda parte da primeira fase de recolha de dados. A lista que resultou dessa parte apresenta-se na Tabela 1.

Ainda na segunda parte procedeu-se à análise da frequência de respostas organizadas por capítulo da ICF e de acordo com as linking rules de Cieza et al. ${ }^{28}$ Tabela 2.

Posteriormente, os conceitos significativos foram submetidos a um processo de correspondência para as várias categorias da classificação da ICF relativas aos fatores ambientais, em um processo de ligação, tal como está esquematizado na Figura 1, correspondendo à terceira parte da primeira fase da coleta de dados.

Uma lista de categorias neutras da ICF foi identificada após análise e transcrição dos conceitos relevantes e organizada com base nos capítulos dos fatores ambientais.

\section{Fase 2}

Essa lista foi enviada aos experts, em conjunto com a lista resultante da segunda parte, isso foi feito para melhor análise e compreensão da informação, com o objetivo de analisarem a informação, reconsiderarem as suas respostas iniciais ou aprovarem os resultados obtidos e apresentados. Estes iriam ser questionados se concordavam ou não com as respostas e, em caso de desacordo, quais alterações sugeriam.

Nessa fase, todos os participantes, de um modo geral, aprovaram os resultados obtidos, não suscitando qualquer alteração digna de registo. Apenas referindo que as informações dos outros participantes completavam as suas, de acordo com as particularidades de cada indivíduo e das suas vivências.

\section{Fase 3}

Nesta fase, o objetivo seria, com base nos resultados obtidos até ao momento, fazer os experts reconsiderarem o conjunto das respostas dadas de forma a chegarem a um consenso. Considerando que na fase anterior não foram verificadas quaisquer modificações que induzissem registo, esta fase não se demonstrou ser necessária pois o consenso grupal foi alcançado na segunda fase.

A análise, interpretação e fundamentação dos resultados obtidos originou a Tabela 3, o produto final deste estudo empírico.

\section{Discussão}

$\infty \times \infty \times \infty \times \infty \times \infty \times \infty \times \infty \times \infty \times \infty \times \infty \times \infty \times \infty \times \infty)$

No que respeita à primeira parte da primeira fase, verificou-se que, para os diferentes participantes, os mesmo fatores poderiam ser facilitadores ou barreiras de acordo com o contexto ou características específicas (tecnologias de apoio, quando são adequadas e atribuídas adequadamente, são um fator facilitador, mas a relação preço/ qualidade/ satisfação, a dificuldade de acesso a elas, as burocracias e o orçamento insuficiente, corresponde a uma barreira).

No que se refere ao domicílio, via e edifícios públicos, tais fatores também são alvo de uma visão binomial e são considerados facilitadores quando se encontram devidamente adaptados (rampas com uma inclinação apropriada, elevadores com dimensões ajustadas, piso antiderrapante ou balcões rebaixados). Quando estes não estão devidamente adaptados (caixas multibanco muito alto, portas duplas ou com molas, passeios danificados e com obstáculos, piso escorregadio, falta de elevadores ou com dimensões desadequadas e rampas com inclinação acentuada ou com localização incorreta) são classificados como barreiras. 0 clima, os transportes públicos, o acesso aos serviços (saúde e educação), o apoio social, as atitudes sociais, também são referidos de acordo com essa dicotomia.

Ao observar as respostas dos vários participantes pôde-se constatar que estas tendem a ser generalistas, não especificando exatamente os contextos ou fatores, o que foi percebido principalmente no acesso à saúde e à educação, ao apoio social, às normas sociais, às práticas e ideologias de apoios ao emprego, ao apoio político e aos serviços de fisioterapia.

Das 142 respostas obtidas, 70 respostas correspondem à identificação de facilitadores e 72 à identificação de barreiras. Essa diferença não é 
muito significativa, revelando que a população já reconhece e valoriza a existência de fatores facilitadores, no entanto, ainda apontam um número considerável de barreiras, que são bastante relevantes para a sua independência e que, portanto, devem ser sempre consideradas. Verifica-se que há mais respostas considerando as tecnologias de apoio como sendo facilitadoras (12 respostas) do que como barreiras (duas respostas). Nos fatores domicílio, via e edifícios públicos verificou-se exatamente o oposto, estes são apontados mais frequentemente como barreiras, quando não se encontram ajustados ou construídos convenientemente (28 respostas), do que como facilitadores (16 respostas).

Os facilitadores mais referidos são as tecnologias de apoio e as barreiras mais apontadas estão nos domicílios, vias e edifícios públicos, devido à falta de adequação aos princípios do desenho universal.

Avaliando a distribuição por capítulo da ICF, observa-se o predomínio de respostas relativas ao primeiro (produtos e tecnologia), com 58 respostas, e ao quinto capítulo (serviços, sistemas e políticas), com 47 respostas, independentemente do fato de serem mencionados como facilitadores ou barreiras. Esses dados revelam maior atenção e predisposição dos utilizadores para avaliar fatores relacionados com tais capítulos, o que acontece por serem aqueles com que os utilizadores de CDR mais tempo lidam e, portanto, os que mais os afetam. Revela-se, assim, que a avaliação e intervenção dos fisioterapeutas na prescrição das tecnologias de apoio e na verificação e identificação de soluções de acessibilidade são fundamentais ${ }^{6,7,17}$.

O elevado número de respostas referentes ao quinto capítulo da ICF é fundamentado pela responsabilidade que se atribui aos serviços, aos sistemas que os regem e às políticas que os orientam, dedicando-se a desenvolver estratégias para reduzir as iniquidades existentes na sociedade ${ }^{26}$ e vincados em documentos formais e orientadores, como é o caso do $1^{\circ}$ Plano de Ação para a Integração das Pessoas com Deficiências ou Incapacidade, publicado pelo Ministério do Trabalho e da Solidariedade Social em 2006.

Ao examinar a terceira parte da primeira fase, respeitando a transcrição dos conceitos relevantes anteriormente analisados, em categorias neutras da ICF observa-se que alguns dos códigos identificados pertencem ao segundo nível e outros são de terceiro nível, dependendo da resposta e da sua indexação às categorias da ICF. As categorias que derivaram das respostas dos participantes não são questionáveis, pois são fruto do consenso do grupo em análise. Procedeuse, dessa forma, à análise das categorias não abordadas.

Perante esta análise verifica-se que há diversas categorias que são referenciadas pela literatura e consideradas indispensáveis para uma avaliação, reavaliação ou para um processo de intervenção holístico a nível comunitário ou individual, que considere as particularidades de cada caso e que tenha como finalidade a sua inclusão. Um dos exemplos, reporta-se aos produtos e às tecnologias gerais para uso pessoal na vida diária (e1150); estes devem ser desenhados ou adaptados às capacidades e necessidades do utilizador ${ }^{6}$, bastando para isso respeitar os princípios do desenho universal.

0 ambiente natural e as mudanças ambientais feitas pelo homem são abordados genericamente, e a literatura sugere que as características do ambiente físico requerem intervenção, conjuntamente com o ambiente cultural, político e social, adquirindo, assim, um papel essencial ${ }^{10,29}$.

Quanto ao papel que o apoio e relacionamentos dos e com os prestadores de cuidados, assistentes pessoais e com os profissionais de saúde (pela relação próxima desenvolvida e pela cooperação necessária), este é considerado um fator cada vez mais discutido na literatura ${ }^{2,18}$. Outros profissionais (e360) não são mencionados, contudo, assumimos aqui a probabilidade de enviesamento de dados, visto que todos os participantes foram utentes de algum serviço de fisioterapia, o que pode ser a razão para a reforçarem.

Concomitantemente, as atitudes são um fator considerado como muito influenciador da funcionalidade ou, pela negativa, da incapacidade e como apresentam-se na sua totalidade ${ }^{6,16}$.

As restantes categorias identificadas pelos participantes são maioritariamente relativas a serviços, havendo apenas uma referência aos 
sistemas relacionados com a área jurídico-legal (e5501). No entanto, é fácil evidenciar medidas políticas e sistemas de saúde, sociais, de transporte e comunicações, potenciadoras, por exemplo, de acessibilidade, de funcionalidade e de inclusão e participação social ${ }^{23,30}$, o que pode revelar falta de sensibilização dos cidadãos em geral e das pessoas com incapacidade na participação ativa relativa aos sistemas e às políticas de saúde, sociais, entre outras.

Embora cientes de algumas limitações deste trabalho, inerentes ao processo de ligação (linking) das respostas para a linguagem e a estrutura da classificação ICF, uma vez que foram de responsabilidade da autora e orientadora deste trabalho, entendemos que, ainda assim, o resultado acrescenta conhecimento ao que existia disponível na literatura.

Um número mais elevado de participantes também aumentaria a probabilidade de se obtermos outras respostas. No entanto, pensamos que considerando as experiências de vida, tempo de evolução na condição e perfil de proatividade dos participantes, a amostra se revelou suficiente, visto que as informações evidenciavam sinais de alguma saturação.

Disponibiliza-se, assim, um checklist para ser utilizado tanto na avaliação como ao longo da intervenção na comunidade ou no domicílio que reflete quais os fatores ambientais identificados pelos utilizadores de CDR devem ser considerados fundamentais. Isto, com a finalidade de avaliar e traçar soluções fora dos componentes tradicionais de abordagem da incapacidade que, simultaneamente, começam a aparecer como elementos-chave em alguns documentos nacionais e internacionais mais atuais, dando real sentido ao modelo biopsicossocial.

0 ponto de vista dos utilizadores de CDR foi muito significativo e discrepante do previsto, revelando que por mais empática que seja a perspectiva dos profissionais, esta nem sempre corresponde às necessidades e aos interesses dessas pessoas. Destaque-se, então, a importância que atribuímos à consulta dos principais interessados.

A mudança do conceito de saúde e o modelo de funcionalidade e incapacidade proposto pela ICF exigem uma atenção progressiva sobre o contexto em que os indivíduos se inserem, demonstrando que fatores ambientais poderão influenciar 0 sucesso na inclusão das pessoas com incapacidade e como devem ser abordados ao longo do processo terapêutico e de capacitação promovido pelo fisioterapeuta.

\section{Considerações finais}

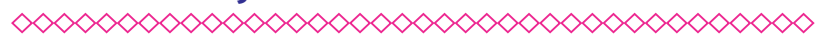

Com este estudo identificaram-se as categorias do componente fatores ambientais da ICF que influenciam a funcionalidade no domicílio e na comunidade/sociedade, de acordo com a percepção dos utilizadores de CDR, sempre que possível confrontada com a literatura existente de forma a conseguir um checklist mais abrangente possivel.

Esta lista é fundamental para a avaliação em fisioterapia, possibilitando a percepção de fatores que podem influenciar e ser influenciados pela fisioterapia, contudo, muitas vezes negligenciados na prática quotidiana. Além disso, permite-nos compreender ou prever relações entre fatores e outros componentes da funcionalidade/incapacidade e, assim, facilitar a intervenção no âmbito da promoção da saúde e bem-estar ou prevenção de condições secundárias à condição atual; facilita a percepção, quer do fisioterapeuta, quer do indivíduo, de quais são as necessidades específicas e reais e, assim, orientar um plano de intervenção apropriado; finalmente, possibilita a adequação de fatores externos ao indivíduo para que este possua máxima funcionalidade e segurança para uma participação ativa e efetiva na sociedade em que se insere.

Por fim, lembramos que a fisioterapia está integrada no capítulo 5 da ICF (serviços, sistemas e políticas), mais especificamente, serviços, sistemas e políticas de prevenção e tratamento de condições de saúde, serviços e programas, controle administrativo, leis, regulamentos e normas que regulam os serviços (e580). A ligação a esse capítulo confere à fisioterapia uma maior diversidade de intervenção, enriquecendo-a. Esse fato impele a um grau de especialização do fisioterapeuta para que este possa intervir em conjunto com outros serviços na comunidade. A finalidade de direcionar as intervenções é a aproximação à realidade contextual dos indivíduos, compreendendo-a e tornando-a apta e facultativa de oportunidades para todas as necessidades. 


\section{Referências}

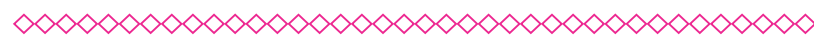

1. WHO. The WHO Family of International Classifications. 2005.

2. WHO. International Classification of Functioning, Disability and Health: ICF. 2001.

3. WHO. The role of environmental factors in functioning and disability- A literature review. 2000.

4. Leonardi, M.; Bickenbach, J.; Ustun, T.; Kostanjsek, N.; Chatterji, S. The definition of disability what is in a name. The Lancet. 2006. Volume 368 (9543): 1219-21.

5. Finger M, Cieza A, Stoll J, Stucki G, Huber E. Identification of Intervention Categories for Physical Therapy, Based on the International Classification of Function, Disability and Health: A Delphi Exercise. Physical Therapy. 2006. Volume 86:1203-20

6. Direcção dos Assuntos Sociais e da Saúde (ResaP). Para a plena cidadania das pessoas com deficiência através de novas tecnologias inclusivas. Conselho da Europa. Estrasburgo. 2002.

7. Keysor J, Jette A, Coster W, Bettger J, Haley S. Association of Environmental Factors with Levels of Home and Community Participation in an Adult Rehabilitation Cohort. American Congress of Rehabilitation Medicine Chicago. 2005.

8. Iwarsson S, Stahl A. Accessibility, usuability and Universal Design- positioning and definition of concepts describing person-environment relationships. Disability Rehabilitation. 2003. Volume 25:57-66.

9. WHO. Estratégia para a reabilitação, a igualdade de oportunidades, a redução da pobreza e a integração social das pessoas com deficiências - Documento de deposição conjunta. 2004.

10. Mont D. Measuring health and disability. The Lancet. 2007. Volume 369:1658-63.
11. Groce N. News, Science and Medicine. Disability in cross-cultural perspective: rethinking disability. The Lancet. 1999. Volume 354:756-7.

12. Haley S. Measuring Physical Disablement: The Contextual Challenge. Physical Therapy. 1994. Volume 74:443-51.

13. Whiteneck G, Fougeyrollas P, Gerhart K. Assessing Medical Rehabilitation Outcomes: the Promise of Outcome Research. Baltimore: Maryland: Paul H Brookes Publishing Co; 1997.

14. McIntosh G, Philips J. Disability Support and Services in Australia. 2002.

15. EUSTAC. Comissão Europeia - DG XII. Educação em Tecnologias de Apoio para Utilizadores Finais - Linhas de Orientação para formadores. 2006.

16. Oliveira R, Silva R, Machado N. As Condições de Acessibilidade e Adequação, para pacientes em cadeira de rodas. Fisioweb. 2006.

17. Meyers A, Anderson J, Miller D, Shipp K, Hoening $\mathrm{H}$. Barriers, facilitators a nd access for wheelchair users: substantive and methodologic lessons from a pilot study of environmental effects. Social Science Medicine. 2002. Volume 55(8):1435- 46.

18. Marmot M. Improvement of social environment to improve health. The Lancet. 1998. Volume 351:57-60.

19. Tomlinson J. Managing maneuverability and rear stability of adjustable manual wheelchairs: an update. Physical Therapy. 2000. Volume 80(9):904-11.

20. Koon N. Design of mobility attachment. National University of Singapore. 2003.

21. Scherer, M.; The change in emphasis from people to person: introduction to the special issue on assistive technology. Disability and Rehabilitation. 2002. Volume 24 (1/2/3): 1-4.

22. WHO. Estratégia para a reabilitação, a igualdade de oportunidades, a redução da pobreza e a integração social das pessoas com deficiências - Documento de deposição conjunta. 2004. 
23. WHO. International Consultation to Review Community-Based Rehabilitation (CBR)Organized by World Health Organization, In collaboration with United Nations Organizations, Non-Governmental Organizations and Disabled People's Organizations. Finlândia. Helsínquia. 2003.

24. WHO. Disability and rehabilitation-WHO action plan 2006-2011. 2005b.

25. Chaves E, Boninger M, Cooper R, Fitzgerald $S$, gray $D$, Cooper R. Assessing the influence of wheelchair technology on perception of participation in spinal cord injury. Physical Medicine Rehabilitation. 2004. Volume 85:1854-8.

26. Ruger J. Ethics of the social determinants of health. The Lancet. 2004. Volume 364:1092-7.

27. Jette A. Toward a Common Language for Function, Disability and Health. Physical Therapy. 2006. Volume 86(5):726-34.

28. Cieza A, Geyh S, Chatterji S, et al. ICF linking rules: an update based on lessons learned. J Rehab Med 2005; 37:212-18.

29. Bent N, Tennant A, Swift T, Posnett J, Scuffham $P$, Chamberiam M. Team approach versus ad hoc health services for young people with disabilities: a retrospective cohort study. The Lancet. 2002. Volume 360:1280-6.

30. Navarro V, Muntaner C, Borrell C, Benach J, Quiroga A, Sanz MR-, et al. Politics and health outcomes. The Lancet. 2006. Volume 368:1033-7. 


\section{Anexos}

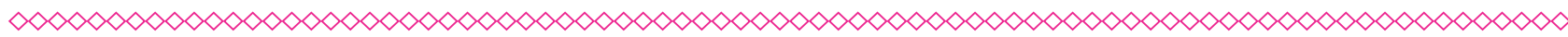

Tabela 1 - Lista de conceitos relevantes resultante das respostas dos participantes com a frequência de respostas para cada conceito

\begin{tabular}{|c|c|c|c|}
\hline \multicolumn{4}{|c|}{$2^{\mathrm{a}}$ Parte } \\
\hline Facilitadores & $\begin{array}{l}N^{\circ} \text { de } \\
\text { respostas }\end{array}$ & Barreiras & $\begin{array}{l}N^{\circ} \text { de } \\
\text { respostas }\end{array}$ \\
\hline $\begin{array}{c}\text { Tecnologia de apoio } \\
\text { adequada (CDR, placas } \\
\text { elevatórias, almofada } \\
\text { antiescara, equipamento do } \\
\text { dia a dia). }\end{array}$ & 12 & $\begin{array}{c}\text { TA com relação preço/qualidade/satisfação do } \\
\text { utilizador fraco }\end{array}$ & 2 \\
\hline $\begin{array}{c}\text { Domicílio adaptado, via e } \\
\text { edifícios públicos acessíveis } \\
\text { (local de trabalho, escola } \\
\text { etc.), rampas com inclinação } \\
\text { suave, elevadores grandes, } \\
\text { piso antiderrapante e balcões } \\
\text { rebaixados. }\end{array}$ & 16 & $\begin{array}{c}\text { Barreiras arquitetônicas no domicílio, via e } \\
\text { edifícios públicos: multibanco muito alto, } \\
\text { portas duplas ou com molas, passeios } \\
\text { danificados e com obstáculos (exemplo: } \\
\text { caixotes do lixo), piso escorregadio, ruas com } \\
\text { piso mal- construído, falta de elevadores ou } \\
\text { elevadores pequenos, rampas muito inclinadas } \\
\text { ou mal-localizadas. }\end{array}$ & 28 \\
\hline Clima (sol) & 3 & Clima (vento, chuva e temperaturas extremas) & 5 \\
\hline Acesso à internet & 2 & $\begin{array}{l}\text { Meio rural, habitações pouco acessíveis, sem } \\
\text { condições sanitárias que não podem ser } \\
\text { utilizadas de forma independente). }\end{array}$ & 2 \\
\hline Transportes públicos & 2 & Transportes públicos & 6 \\
\hline $\begin{array}{l}\text { Transporte particular } \\
\text { adaptado }\end{array}$ & 7 & $\begin{array}{c}\text { Falta de civismo (comportamentos como } \\
\text { estacionar no passeio ou em locais próprios } \\
\text { para pessoas com incapacidade) }\end{array}$ & 6 \\
\hline $\begin{array}{c}\text { Apoio da família, amigos e } \\
\text { companheiro(a) }\end{array}$ & 9 & $\begin{array}{c}\text { Dificuldade no acesso a TA (burocracias e } \\
\text { orçamento insuficiente) }\end{array}$ & 1 \\
\hline Benefícios fiscais & 2 & Falta de formação & 2 \\
\hline Acesso à saúde e à educação & 4 & Acesso à educação e à saúde & 2 \\
\hline $\begin{array}{l}\text { Apoio ao emprego } \\
\text { (empresas) }\end{array}$ & 2 & \multirow{2}{*}{$\begin{array}{l}\text { Falta de apoio do Estado, pouco controle da } \\
\text { legislação. }\end{array}$} & \multirow[t]{2}{*}{3} \\
\hline Apoio político & 1 & & \\
\hline Apoio social & 3 & Falta de apoio social & 4 \\
\hline $\begin{array}{c}\text { Atitudes sociais (mentalidade } \\
\text { aberta, sensibilização para a } \\
\text { incapacidade }\end{array}$ & 3 & $\begin{array}{l}\text { Atitudes ('coitadinho', mentalidade fechada, } \\
\text { pensar que utilizadores de CDR não têm } \\
\text { qualificação para um trabalho específico) }\end{array}$ & 8 \\
\hline $\begin{array}{c}\text { Exercício físico ou atividade } \\
\text { desportiva }\end{array}$ & 1 & Normas sociais & 1 \\
\hline Serviços (Fisioterapia) & 2 & Práticas e ideologias & 1 \\
\hline $\begin{array}{l}\text { Prioridade de atendimento } \\
\text { em certos serviços públicos }\end{array}$ & 1 & Serviços, sistemas e políticas & 1 \\
\hline Total & 70 & & 72 \\
\hline \multicolumn{3}{|c|}{ Total absoluto } & 142 \\
\hline
\end{tabular}


Tabela 2 - Número de respostas por capítulo dos fatores ambientais

\begin{tabular}{|c|c|}
\hline Capítulo dos fatores ambientais & Número de respostas \\
\hline Produtos e tecnologia & 58 \\
\hline Ambiente natural e mudanças ambientais feitas pelo homem & 8 \\
\hline Apoio e relacionamentos & 16 \\
\hline Atitudes & 13 \\
\hline Serviços, sistemas e políticas & 47 \\
\hline Total de respostas & 142 \\
\hline
\end{tabular}

Figura 1 - Esquema do processo de ligação para as várias categorias de fatores ambientais da classificação da ICF

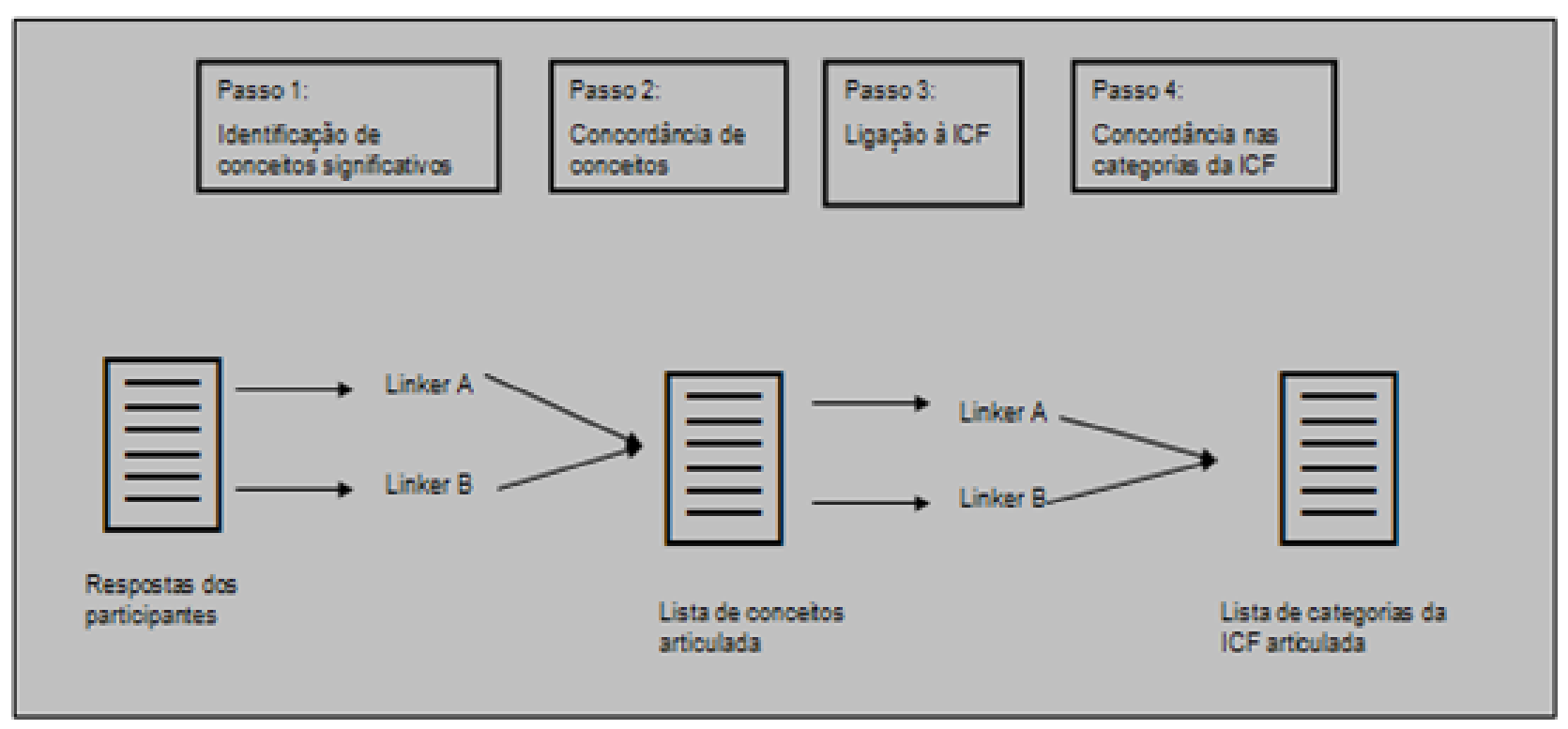

(clique para voltar ao texto) 


\section{Tabela 3 - Checklist dos fatores que influenciam a funcionalidade dos utilizadores de cadeira de rodas}

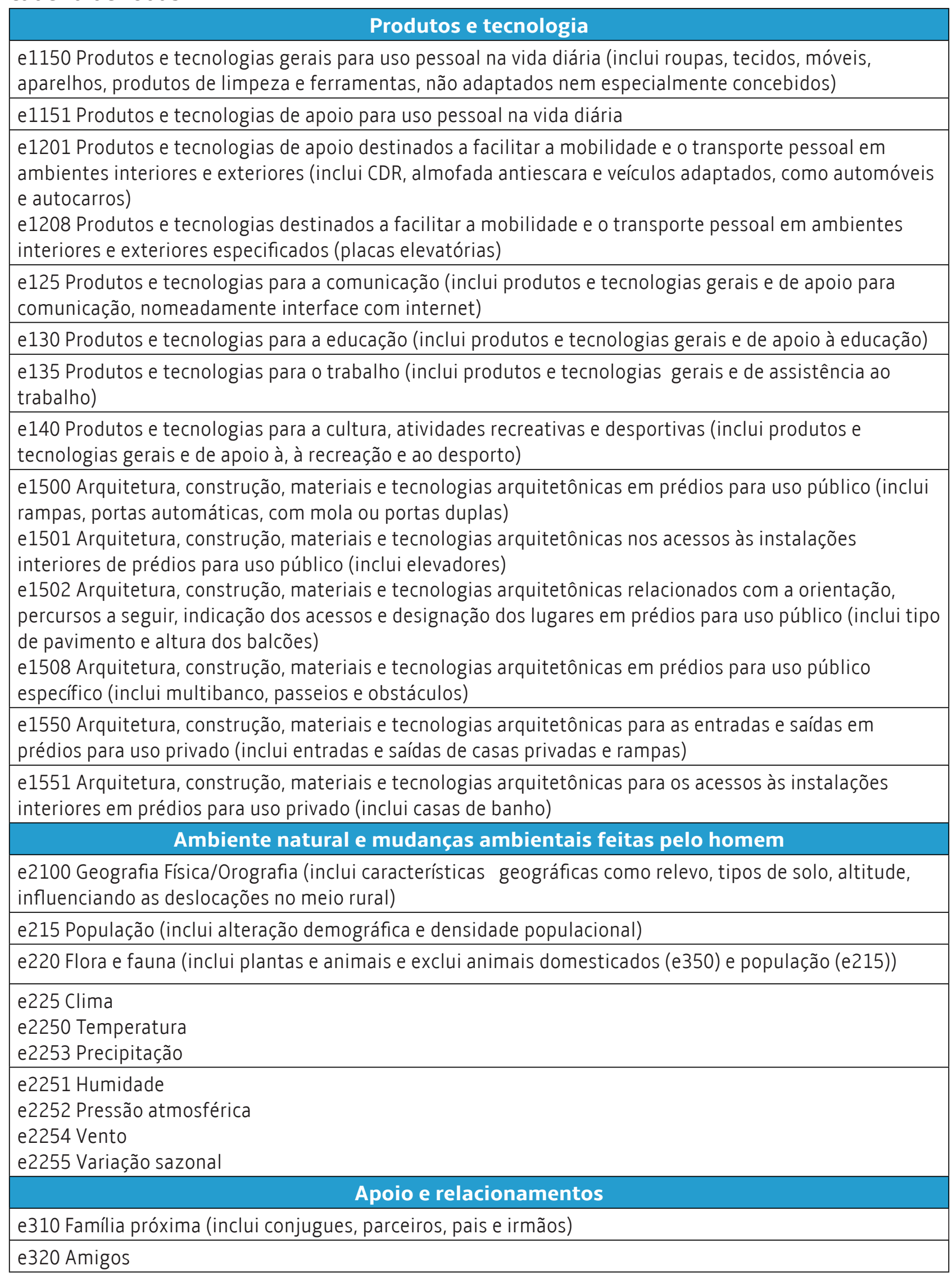




\begin{tabular}{|l|}
\hline e325 Conhecidos, pares, colegas, vizinhos e membros da comunidade (inclui vizinhos) \\
\hline e330 Pessoas em posição de autoridade (inclui decisores) \\
\hline e340 Prestadores de cuidados pessoais e assistentes pessoais \\
\hline e355 Profissionais de saúde \\
\hline e360 Outros profissionais
\end{tabular}

\section{Atitudes}

e410 Atitudes individuais de membros da família próxima (inclui opiniões e crenças gerais ou específicas de membros familiares próximos sobre a pessoa ou sobre outras questões que influenciem o comportamento)

e415 Atitudes individuais de membros da família alargada

e420 Atitudes individuais dos amigos

e425 Atitudes individuais de conhecidos, pares, colegas, vizinhos e membros da comunidade

e430 Atitudes individuais de pessoas em posições de autoridade

e435 Atitudes individuais de pessoas em posições subordinadas

e440 Atitudes individuais de prestadores de cuidados pessoais e assistentes pessoais

e445 Atitudes individuais de estranhos

e450 Atitudes individuais de profissionais de saúde

e455 Atitudes individuais de outros profissionais

e460 Atitudes sociais (inclui opiniões e crenças gerais ou específicas mantidas em geral pelas pessoas de uma cultura, sociedade, agrupamentos subculturais ou outros grupos sociais, sobre outros indivíduos ou sobre outras questões sociais, políticas e económicas que influenciam o comportamento e as ações dos indivíduos ou dos grupos)

e465 Normas, práticas e ideologias sociais (inclui costumes, práticas, regras e sistemas abstratos de valores e crenças normativas que surgem dentro dos contextos sociais e que afetam ou criam práticas e comportamentos sociais e individuais, tais como, normas sociais de moral, etiqueta e comportamento religioso, doutrina religiosa e normas e práticas resultantes e normas que regulam os rituais ou as reuniões sociais)

\section{Serviços, sistemas e políticas}

e510 Serviços, sistemas e políticas relacionados com a produção de bens de consumo (que regulam e que são responsáveis pela produção de objetos e produtos consumidos ou utilizados pelas pessoas)

e515 Serviços, sistemas e políticas relacionados com a arquitetura e a construção (relacionados com o projeto e a construção de edifícios, públicos e privados)

e520 Serviços, sistemas e políticas relacionados com o planeamento de espaços abertos (relacionados com o planeamento, projeto, criação e manutenção de áreas públicas e de áreas privadas nas zonas rurais, residenciais e urbanas)

e525 Serviços, sistemas e políticas relacionados com a habitação (que proporcionam abrigo, habitação e alojamento para as pessoas)

e530 Serviços, sistemas e políticas relacionados com os serviços de utilidade pública e5350 Serviços relacionados com a comunicação(inclui serviços e programas orientados para a transmissão de informações por meio de vários métodos que inclui os serviços de acesso à internet e a telefones adaptados) e5351 Sistemas relacionados com a comunicação (inclui mecanismos de controle administrativo e de supervisão)

e5352 Políticas relacionados com a comunicação (inclui leis, regulamentos que regulam a transmissão de informação por meio de vários métodos) 
e5400 Serviços relacionados com os transportes (inclui serviços e programas orientados para a deslocação de pessoas utilizando transportes públicos)

e5401 Sistemas relacionados com os transportes (mecanismos de controle administrativo e de supervisão que regulam a deslocação de pessoas)

e5402 Políticas relacionadas com os transportes (leis, regulamentos e normas que regulam a deslocação de pessoas)

e545 Serviços, sistemas e políticas relacionados com a proteção civil (orientados para a proteção das pessoas e das propriedades)

e5500 Serviços relacionados com a área jurídico-legal (serviços e programas orientados para o estabelecimento da autoridade do Estado)

e5501 Sistemas relacionados com a área jurídico-legal (mecanismos de controle administrativo e de supervisão que regulam a administração da justiça, tais como, sistemas para o desenvolvimento e supervisão de regras formais)

e5502 Políticas relacionados com a área jurídico-legal (leis, regulamentos e normas que regem a administração da justiça)

e555 Serviços, sistemas e políticas relacionados com associações e organizações (relacionados com grupos de pessoas que se organizaram para a prossecução de interesses comuns e não comerciais, com frequência numa estrutura tipo associação com membros inscritos)

e5600 Serviços relacionados com os meios de comunicação (orientados para assegurar a comunicação em massa utilizando meios como a televisão e a internet)

e5601 Sistemas relacionados com os meios de comunicação (mecanismos de controle administrativo e de supervisão que regulam o fornecimento de notícias e informações ao público em geral)

e5602 Políticas relacionadas com os meios de comunicação (leis, regulamentos e normas que regulam o fornecimento de notícias e informações ao público em geral)

e565 Serviços, sistemas e políticas relacionados com a economia (relacionados com o sistema geral de produção, distribuição, consumo e utilização de bens e serviços)

e5700 Serviços relacionados com a segurança social (serviços e programas que assegurem a atribuição de apoio econômico para pessoas que têm necessidades de usufruir de assistência pública financiada pelas receitas fiscais ou por esquemas de contribuição)

e5701 Sistemas relacionados com a segurança social (mecanismos de controle administrativo e de supervisão que regulam os programas e esquemas que asseguram a atribuição de apoio econômico ) e5702 Políticas relacionados com a segurança social (leis, regulamentos e normas que regulam os programas e esquemas que asseguram a atribuição de apoio econômico )

e575 Serviços, sistemas e políticas relacionados com o apoio social em geral (orientados para dar apoio àqueles que necessitam de ajuda)

e5800 Serviços relacionados com a saúde (serviços e programas de nível local e comunitário que têm por finalidade proporcionar intervenções junto dos indivíduos para o seu bem-estar)

e5801 Sistemas relacionados com a saúde (mecanismos de controle administrativo e de supervisão que regulam a gama de serviços fornecidos aos indivíduos)

e5802 Políticas relacionados com a saúde (leis, regulamentos e normas que regulam a gama de serviços disponíveis)

e5850 Serviços relacionados com a educação e a formação profissional (serviços e programas, orientados para a educação e a aquisição, manutenção e melhoria de conhecimentos e de competências em áreas de especialidades profissionais)

e5851 Sistemas relacionados com a educação e a formação profissional (mecanismos de controle administrativo e de supervisão que regulam a oferta de programas educativos) 
e5852 Políticas relacionados com a educação e a formação profissional (leis, regulamentos e normas que regulam a administração de programas educativos)

e5900 Serviços relacionados com o trabalho e o emprego (serviços e programas que têm por finalidade encontrar trabalho adequado para pessoas desempregadas ou que procuram um trabalho diferente ou para dar apoio a indivíduos já empregados)

e5901 Sistemas relacionados com o trabalho e o emprego (mecanismos de controle administrativo e de supervisão organizativa que regulam a distribuição do trabalho e de outras ocupações remuneradas)

e5902 Políticas relacionados com o trabalho e o emprego (leis, regulamentos e normas que regulam a distribuição do trabalho e de outras ocupações remuneradas)

e595 Serviços, sistemas e políticas relacionados com o sistema político (relacionados com sistema de votação, eleições e governo) 